Chrischta Ganz

Die heutige Situation aus der Sicht einer Naturheilpraktikerin

Die Situation der Schweizer Naturheilpraktiker und Naturheilpraktikerinnen ist bis heute kompliziert und uneinheitlich: Die Studierenden absolvieren nach einigen Jahren Ausbildung die Abschlussprüfungen an der jeweiligen Schule. Danach müssen sie sich in manchen Kantonen zusätzlich einer kantonalen Prüfung unterziehen und/oder sich um eine Berufsausübungsbewilligung bemühen. Zusätzlich sind sie aufgefordert, ihre Fachkompetenz bei verschiedenen privaten Institutionen zu registrieren. Selbstverständlich fordern auch Verbandsmitgliedschaften Kompetenznachweise.

Die Komplementärtherapeuten und Komplementärtherapeutinnen befinden sich in einer ähnlich komplizierten Situation.

Die Kernprobleme der praktizierenden Naturheilpraktiker und Komplementärtherapeuten sind [1]:

- das uneinheitliche Ausbildungsund Abschlussniveau;

- keine offizielle Berufsanerkennung;

- kein offizieller Platz im Gesundheitswesen;

- die fehlende Transparenz für Bevölkerung und Patienten;

- die uneinheitlichen Berufsbewilligungen in Kantonen;

- die uneinheitliche Abgabekompetenz für Heilmittel durch unterschiedliche kantonale Gesetzgebungen;

- die kostenpflichtige und aufwendige Registrierung bei ASCA (Stiftung zur Anerkennung und Entwicklung der Alternativ- und

\title{
Die Höhere Fachprüfung (HFP) der Schweizer Naturheilpraktiker und Komplementärtherapeuten aus der Sicht einer Naturheilpraktikerin
}

Komplementärmedizin), beim EMR (Erfahrungsmedizinisches Register), bei SPAK (Qualitätssicherungsstelle des NVS) und bei den Krankenkassen.

\section{Der Berufsbildungsprozess bei den Naturheilpraktikern}

In Kooperation mit dem Staatssekretariat für Bildung, Forschung und Innovation (SBFI; vormals zuständiges Bundesamt für Berufsbildung (BBT)) erarbeitet die OdA AM zurzeit die Berufsreglementierung. Die normativen Prozesse sind in vollem Gange. Das Projekt steht kurz vor dem Ziel, einen neuen, reglementierten Berufsabschluss der Alternativmedizin zu schaffen. Mit der Reglementierung werden die Therapeuten der Alternativmedizin offiziell in das schweizerische Gesundheitswesen integriert. Neben der Definition eines einheitlichen Berufsbildes mit entsprechenden Handlungskompetenzen wurden die Ausbildungsvorgaben und -ziele formuliert, die Qualifikationsverfahren für die Handlungskompetenzen sichergestellt und die Validierungsverfahren für bereits Praktizierende sowie zur Qualitätssicherung entwickelt [2]. Integrale Bestandteile des neuen Berufsbildes sind die medizinische Einschätzung sowie die fachrichtungsspezifische Diagnose, die Empfehlung, Anwendung und Abgabe von nicht verschreibungspflichtigen Heilmitteln, die Behandlung von akuten und chronischen Krankheiten auf der Basis von alternativmedizinischen Gesamtkonzepten sowie invasive Behandlungen.

Die Kompetenzen des Naturheilpraktikers werden weiter gefasst als diejenigen des Komplementärtherapeuten, was eine tiefer greifende Ausbildung und Abschlussprüfung bedingt.

$\mathrm{Zu}$ den Kernkompetenzen zählen das alternativmedizinische Handeln und die Gestaltung therapierelevanter Beziehungen.

Mit «alternativmedizinischem Handeln» ist in der Berufspolitik gemeint:

- Therapieprozesse mittels eines alternativmedizinischen Gesamtsystems gestalten;

- Patienten in unterschiedlichen Krankheitsphasen nach alternativmedizinischen Grundsätzen betreuen;

- Patientenressourcen sowie ihre Gesundheitskompetenz stärken.

Mit der «Gestaltung therapierelevanter Beziehungen» ist in der Berufspolitik gemeint:

- Beziehungen mit Patienten und Bezugspersonen fallbezogen gestalten;

- Beziehungen mit Fachpersonen zur Gewährleistung der Patientenführung nach alternativmedizinischen Grundsätzen gestalten.

Die Kernkompetenzen bilden die berufliche Hauptaktivität, d.h. die therapeutische Arbeit in der naturheilkundlichen Praxis. Hier gibt es keine Durchlässigkeit zu anderen Gesundheitsberufen.

Als unterstützende Kompetenzen werden Gesundheit entwickeln und
(๑) 2014 S. Karger GmbH, Freiburg 


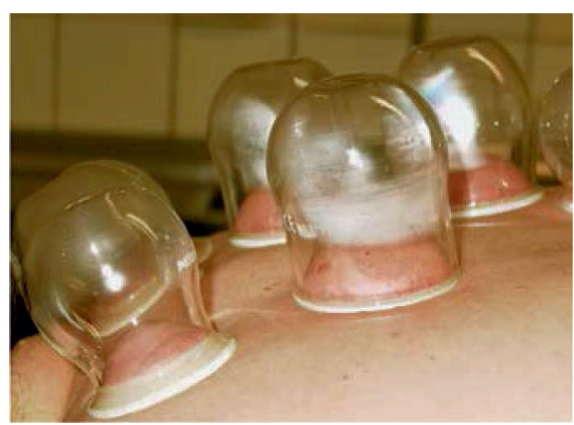

Abb. 1. Schröpfbehandlung, wie sie in der Alternativmedizin angewendet wird.

fördern, lernen und lehren, sich professionell verhalten, sich vernetzen/im Team arbeiten und einen Betrieb führen definiert. Die unterstützenden Kompetenzen machen nur etwa 10 $20 \%$ der beruflichen Aktivität aus und haben grosse Durchlässigkeit gegenüber anderen Gesundheitsberufen.

Unabhängig von der Fachrichtung (Traditionelle Chinesische Medizin (TCM), Traditionelle Europäische Naturheilkunde (TEN), Homöopathie, Ayurveda) (Abb. 1) ist der Aufbau der Ausbildung und der Prüfung (Prüfungsteile, Bedingungen, Zulassung usw.) identisch. Nach der schulmedizinischen Grundausbildung (M1), der Ausbildung in der gewählten Fachrichtung (M2), in Gesundheit/ Ethik (M3), in der Arbeit als Therapeut/in (M4), in Betrieb führen und managen (M5) sowie der praktischen Ausbildung (M6) ist ein Praktikum Berufspraxis unter Mentorat (M7) zu absolvieren, um für die HFP zugelassen zu werden.

Für bereits Praktizierende ist eine direkte Zulassung an die HFP (nach 5 Jahren Berufspraxis) bzw. eine erleichterte Prüfung (nach 10 Jahren Berufspraxis nur P1 und P2) möglich.

Im Frühling dieses Jahres fanden in Sursee die Pilotprüfungen in den Fachrichtungen der Alternativmedizin statt. Es war eine intensive Woche, die von allen Beteiligten höchsten Einsatz verlangte. Die vier Prüfungsteile der HFP sollen an dieser Stelle vorgestellt werden [1].
P1: Schriftliche Fallstudie, in der ein Patientenfall dokumentiert, begründet und vorgestellt wird (vorgängig, schriftlich)

- Patientenfall: Behandlungs- und Betreuungsprozesse eines Patienten mit einer Krankheit von chronisch wiederkehrendem Charakter, beginnend mit der ersten Kontaktnahme sowie der Erst- und Folgeanamnesen (relevante Überlegungen, Problemstellungen, Erkrankungs- und Gesundungsprozesse, Abläufe und Therapieplanung);

- Darlegung der Stärkung der Patientenressourcen und Förderung der Gesundheitskompetenzen;

- Ausrichtung auf das jeweilige fach(richtungs-)spezifische Denken, Handeln und Zielsetzen (gemäss Berufsbild AM).

\section{P2: Fachgespräch zum Inhalt \\ der erstellten Fallstudie P1 (mündlich, Dauer $45 \mathrm{~min}$ )}

1. Kurzvorstellung des Falls mit Fakten für Gesundheitsfachpersonen;

2. Fachgespräch mit Fragen zum vorgestellten Fall und folgenden Aspekten:

- Nachvollziehbarkeit der Begründungen des jeweiligen Handelns und Umsetzung der Arbeitsprozesse;

- fachliche Korrektheit und Bezugnahme zu Prinzipien der Fachrichtung;

- Verständnis und Umsetzung der beruflichen Rolle(n).

\section{P3: Fallbearbeitung von zwei} schriftlichen Patientenfällen

(mündlich und praktisch,

Dauer $150 \mathrm{~min}$ )

Der Kandidat bzw. die Kandidatin erhält zwei Papierfälle, die Fallinformationen mit Lücken enthalten; Teilinformationen werden auf Nachfrage gegeben.

In drei Phasen wird die Einschätzung der Fälle zu schulmedizinischen und fachrichtungsrelevanten Fragestellungen geprüft:

- Ersteinschätzung: Wie ist die Ersteinschätzung und erste Informa tionsbeschaffung?

- Analyse: Wie wird recherchiert und wie werden erste Hypothesen erweitert, verändert oder weiter verfolgt?

- Präsentation und Fachgespräch mit den Experten: Wie ist die Schlussanalyse und wie sieht die Behandlungsplanung aus?

P4: Behandlung eines/einer LivePatienten/Patientin (praktisch und mündlich, Dauer max. 180 min)

Der vierte Prüfungsteil teilt sich in drei Teile:

1. Anamnese, Befunderhebung;

2. Analyse, Auswertung, Therapiebeginn;

3. Präsentation der Analyse und Fachgespräch mit den Experten.

Warum soll ein/e bereits praktizierende/r Naturheilpraktiker/in das Diplom machen?

Was der HFP-Abschluss aus der Sicht der Autorin mit sich bringt, ist eine klare Abgrenzung gegenüber der Esoterik, wo - vielfach missverständlich und meist missverstanden - der Beruf des Naturheilpraktikers abgewertet wird. Diesbezüglich ist der politische Berufsbildungsprozess sehr wichtig. Die Naturheilkunde ist eine «andere Medizin», die im schweizerischen Gesundheitssystem und der Berufspolitik schon lange ihren Platz hat, aber nun auch rechtlich verankert und definiert wird. Die grosse Gabe der Naturheilkunde ist die individuelle und ganzheitliche Sicht auf den Menschen - eben nicht «das Knie in Zimmer 367», sondern Herr Meier mit seinem aktuellen Leiden und seinen konstitutionellen Grundlagen, seiner Geschichte, seiner momentanen Verfassung und seinen Zukunftsvorstellungen, eingebunden in sein soziales Umfeld. 


\section{Ausblick}

Geplant ist bis Oktober 2014 die Inkraftsetzung und Ausschreibung der ersten HFP AM und im März 2015 die Durchführung der ersten regulären HFP AM [1]. Auch die erste HFP KT ist für Frühling 2015 vorgesehen. Weiterführende Informationen können auf der Internetseite der OdA-AM bzw. der OdA-KT eingesehen werden [2].

\section{Situation in den deutschsprachigen Nachbarländern}

In Deutschland sieht die Situation rechtlich anders aus. Das deutsche
Heilpraktikergesetz ist eigentlich gar kein «Zulassungsgesetz» und hat eine «schwierige Geschichte», da es eigentlich aus dem Dritten Reich stammt. Weil der konkrete Praxisalltag den deutschen Heilpraktikern vieles erlaubt, wird von den meisten naturheilkundlichen Fachpersonen die Haltung «Finger weg vom Heilpraktikergesetz» vertreten [3]. Interessant ist die Tatsache, dass die Schweizer sich meist mit «Naturheilpraktiker/ innen», ihre deutschen Berufskollegen sich hingegen als «Heilpraktiker/innen» bezeichnen. Auch dies ist kulturell-historisch bedingt.

In Österreich gibt es offiziell keine Naturheilpraktiker/innen. Nur akademisch ausgebildeten Ärzten und
Ärztinnen ist die Ausübung der Heilkunde gestattet. Allerdings gilt die traditionelle Naturheilkunde in Österreich als immaterielles UnescoKulturgut [4].

\section{Literatur}

1 QSK Infoveranstaltung HFP. Mai 2014, Olten.

2 www.oda-am.ch/www.oda-kt.ch.

3 Liebaus KF: Die Zukunft entwickelt sich stets aus der Gegenwart. Deutsche Heilpraktikerzeitschrift 2013;8:64-74.

4 Bertschi-Stahl HD: Im Dschungel der Heilberufe wird aufgeräumt. Deutsche Heilpraktikerzeitschrift 2013;8:62-64. 